\title{
Predictions from Lattice QCD
}

\author{
A.S. Kronfeld ${ }^{*}, a$, I.F. Allison ${ }^{b}$, C. Aubin ${ }^{c, d}$, C. Bernard ${ }^{d}$, C.T.H. Davies ${ }^{b}$, C. DeTar ${ }^{e}$, \\ M. Di Pierro ${ }^{f}$, E.D. Freeland ${ }^{g}$, Steven Gottlieb ${ }^{h}$, A. Gray ${ }^{i}$, E. Gregory ${ }^{j}$, U.M. Heller ${ }^{k}$, \\ J.E. Hetrick ${ }^{l}$, A.X. El-Khadra ${ }^{m}$, L. Levkova ${ }^{h}$, P.B. Mackenzie ${ }^{a}$, F. Maresca ${ }^{e}$, \\ D. Menscher ${ }^{m}$, M. Nobes ${ }^{n, o}$, M. Okamoto ${ }^{a}$, M.B. Oktay ${ }^{m}$, J. Osborn $^{e}$, D. Renner ${ }^{j}$, \\ J.N. Simone ${ }^{a}$, R. Sugar ${ }^{p}$, D. Toussaint ${ }^{j}$, H.D. Trottier $^{o}$
}

E-mail: ask@fnal.gov

${ }^{a}$ Fermi National Accelerator Laboratory, Batavia, Illinois 60510, USA

${ }^{b}$ Department of Physics and Astronomy, Glasgow University, Glasgow, Scotland G12 8QQ, UK

${ }^{c}$ Physics Department, Columbia University, New York, New York 10027, USA

${ }^{d}$ Department of Physics, Washington University, St. Louis, Missouri 63130, USA

${ }^{e}$ Physics Department, University of Utah, Salt Lake City, Utah 84112, USA

${ }^{f}$ School of Computer Science, Telecommunications and Information Systems, DePaul University,

Chicago, Illinois 60604, USA

${ }^{g}$ Liberal Arts Department, School of the Art Institute, Chicago, Illinois 60603, USA

${ }^{h}$ Department of Physics, Indiana University, Bloomington, Indiana 47405, USA

${ }^{i}$ Department of Physics, The Ohio State University, Columbus, Ohio 43210, USA

${ }^{j}$ Department of Physics, University of Arizona, Tucson, Arizona 85721, USA

${ }^{k}$ American Physical Society, Ridge, New York 11961, USA

${ }^{l}$ Physics Department, University of the Pacific, Stockton, California 95211, USA

${ }^{m}$ Physics Department, University of Illinois, Urbana, Illinois 61801, USA

${ }^{n}$ Laboratory of Elementary-Particle Physics, Cornell University, Ithaca, New York 14853, USA

${ }^{o}$ Physics Department, Simon Fraser University, Burnaby, British Columbia V5A 1S6, Canada

${ }^{p}$ Department of Physics, University of California, Santa Barbara, California 93106, USA

\section{Fermilab Lattice, MILC, and HPQCD Collaborations}

In the past year, we calculated with lattice QCD three quantities that were unknown or poorly known. They are the $q^{2}$ dependence of the form factor in semileptonic $D \rightarrow K l v$ decay, the decay constant of the $D$ meson, and the mass of the $B_{c}$ meson. In this talk, we summarize these calculations, with emphasis on their (subsequent) confirmation by experiments.

XXIIIrd International Symposium on Lattice Field Theory

25-30 July 2005

Trinity College, Dublin, Ireland

\footnotetext{
*Speaker.
} 


\section{Introduction and Background}

In recent years, lattice QCD has reached the stage where many calculations of hadron masses, mass splittings, and operator matrix elements agree with experimental measurements. The key has been the inclusion of sea quarks. The progress has been especially striking [1] when the light quarks (sea and valence) are implemented as staggered quarks, with an improved action.

Some of the ingredients of these calculations are controversial. Staggered quarks come in four tastes, three of which must be removed to obtain each individual flavor. For sea quarks, this is done by taking the fourth root of the fermion determinant; for valence quarks, by projecting onto the desired taste sector. Furthermore chiral perturbation theory must be modified [2]. Although evidence for the validity of these "tricks" is slowly accumulating, a proof remains at large [3]. In addition, much of the success of Ref. [1] comes from hadrons with heavy quarks. Although debate on heavy quarks in lattice QCD seems to have subsided, checks are still useful.

In this paper, we discuss three calculations, with emphasis on their subsequent experimental confirmation. They are the normalization and $q^{2}$-dependence of the $D \rightarrow K l v$ form factor; the decay constants of the $D^{+}$and $D_{s}$ mesons; and the mass of the $B_{c}$ meson. Each tests a somewhat different combination of the ingredients, and the following table gives an informal guide:

\begin{tabular}{c|ccc}
\hline \hline calculation & light sea & light valence & heavy \\
\hline semileptonic $f_{+}\left(q^{2}\right)$ & $\star \star$ & $\star \star$ & $\star \star$ \\
leptonic $f_{D}$ & $\star \star$ & $\star \star \star$ & $\star \star$ \\
$B_{c}$ mass & $\star \star$ & - & $\star \star \star$ \\
\hline \hline
\end{tabular}

The chiral extrapolation, which is more sensitive to valence quarks than sea quarks, turned out to be more important for the decay constant than the form factor. The $B_{c}$ meson has no light valence quarks at all, but one should expect an accurate calculation only if heavy-quark discretization effects are under control.

Successful predictions are, of course, not a substitute for a proof. They are still useful. Even if the experts are confident of all the elements of their numerical calculations, non-experts are interested in an end-to-end check [4]. The quantities discussed here are ideal candidates: they are straightforward to compute; the first "good" experimental measurements were not expected until this year; and new physics is unlikely to contribute significantly.

\section{Semileptonic D Decays}

Semileptonic decays such as $D \rightarrow K l v$ are mediated by electroweak vector currents. The matrix element $\left\langle K\left|V^{\mu}\right| D\right\rangle$ is parametrized by form factors. For a vector current there are two, but experimentally only the one called $f_{+}\left(q^{2}\right)$ is accessible; the rate from the other one, $f_{0}\left(q^{2}\right)$, is suppressed by $m_{l}^{2}$. Here $q^{2}$ is the momentum transferred to the lepton-neutrino system, falling in the range $0 \leq q^{2} \leq q_{\max }^{2}=\left(m_{D}-m_{K}\right)^{2}$. In lattice QCD, discretization effects are smallest when the momentum $\boldsymbol{p}$ of the kaon is small, and then $q^{2}$ is not too far from $q_{\max }^{2}$.

Experiments usually measure the branching fraction and quote the normalization $f_{+}(0)$, after making assumptions about the $q^{2}$ dependence. While our results were still preliminary [5], experimental results came out for the normalization of $D \rightarrow K l v[6]$ and $D \rightarrow \pi l v$ [7]. The agreement 
with our final results [8] is excellent. For example, we find $f_{+}^{D \rightarrow K}(0)=0.73(3)(7)$ [8] while BES measures $f_{+}^{D \rightarrow K}(0)=0.78(5)$ [6]. Our calculations of the normalization are also consistent with the soft pion theorem, which states $f_{0}\left(q_{\max }^{2}\right)=f_{D} / f_{\pi}$.

In principle, the shape of the form factors can be computed directly in lattice QCD. In practice, we calculated at a few values of $\boldsymbol{p}$ and used the Bećirević-Kaidalov (BK) form [9] to fix the full $q^{2}$ dependence of $f_{+}$and $f_{0}$. Then the normalization of $f_{+}$comes mainly from $f_{0}$ through a kinematic constraint $f_{+}(0)=f_{0}(0)$. The BK Ansatz and calculations near $q_{\max }^{2}$ determine the shape. It was important, therefore, to measure the $q^{2}$ dependence experimentally. In photoproduction of charm off fixed nuclear targets, the FOCUS Collaboration was able to collect high enough statistics to trace out the $q^{2}$ distribution of the decay [10]. This setup does not yield an absolutely normalized branching ratio, so one is left to compare $f_{+}\left(q^{2}\right) / f_{+}(0)$.

In Fig. 1 we plot our result for $f_{+}\left(q^{2}\right) / f_{+}(0)$ vs. $q^{2} / m_{D_{s}^{*}}^{2}$. The errors from $f_{+}(0)$ must be propagated to non-zero $q^{2}$, so for $f_{+}\left(q^{2}\right) / f_{+}(0)$ the errors grow with $q^{2}$. Figure 1 shows $1-\sigma$ bands of statistical (orange) and all uncertainties (yellow) added in quadrature [11]. As one can see, the $q^{2}$ dependence of lattice QCD (curve and error band) and experiment (points) agree excellently, although the uncertainties are still several per cent.

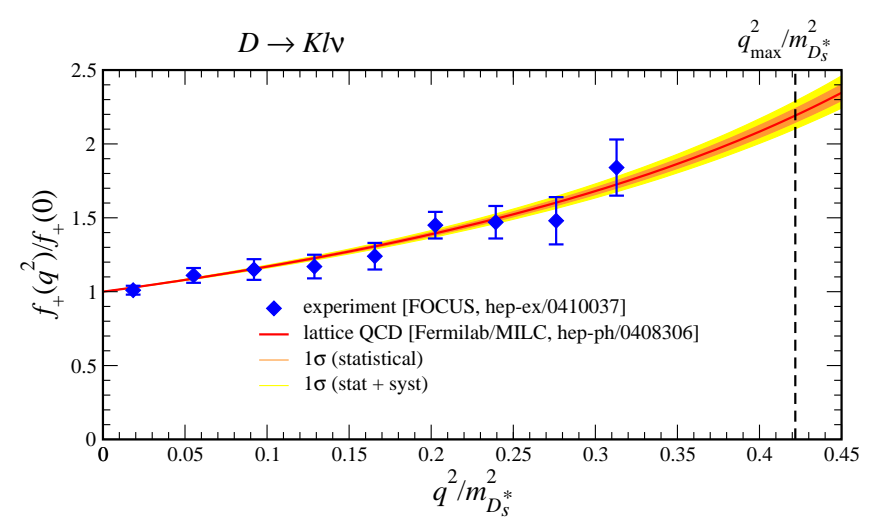

Figure 1: Shape of form factor $f_{+}\left(q^{2}\right) / f_{+}(0)$ vs. $q^{2} / m_{D_{s}^{*}}^{2}$, compared with experiment [10].

\section{Leptonic $D$ Decays}

We also computed the hadronic matrix element for the leptonic decay of charmed mesons, $f_{D^{+}}$ and $f_{D_{s}}$. The first (experimental) measurements of $f_{D^{+}}$appeared in 2004, with three events from BES [12] and eight from CLEO [13]. Neither provides a stringent test of QCD, but CLEO- $c$ was just starting its run and promised 5-8 times higher statistics by the Summer 2005 Lepton-Photon Symposium [4]. At Lattice 2004 [14], we presented preliminary results for $f_{D^{+}}$, based on one lattice spacing, $a \approx 0.125 \mathrm{fm}$. Our aim was to extend the running to two other lattice spacings and, of course, to improve our understanding of other aspects of the calculation, such as the chiral extrapolation. Details are given in the ensuing publication [15]. We find

$$
f_{D^{+}}=201 \pm 3 \pm 17 \mathrm{MeV}
$$

where the first error is from finite Monte Carlo statistics, the second is a sum in quadrature of several systematics. A conservative (but not naïve) estimate of heavy-quark discretizations effects, 
as discussed in Ref. [16], is the second largest (largest) systematic on $f_{D^{+}}\left(f_{D_{s}}\right)$. A few days after our paper was posted on the arXiv, CLEO- $c$ announced its new measurement [17]

$$
f_{D^{+}}=223 \pm 17 \pm 3 \mathrm{MeV},
$$

based on $47 \pm 8$ events. At the 1- $\sigma$ level, the agreement between Eqs. (3.1) and (3.2) is fine. One should keep in mind that the experiment actually determines $\left|V_{c d}\right| f_{D^{+}}$. CLEO-c [17] assumes that $\left|V_{c d}\right|=\left|V_{u s}\right|$ and uses a recent average of $\left|V_{u s}\right|$ from semileptonic $K$ decay.

It is interesting to look at the $n_{f}$ dependence of $f_{D_{s}}$, shown in Fig. 2(a). Of course, quenched results vary widely, but we show one [18] carried out with similar choices for heavy quarks, renormalization factors, etc. One sees a trend of $f_{D_{s}}$ to increase with $n_{f}$. A similar comparison of $f_{D^{+}}$, in Fig. 2(b), is less instructive, because the chiral extrapolations in Refs. [18, 19] started at large quark masses and are, hence, less reliable than in the present work.

(a)

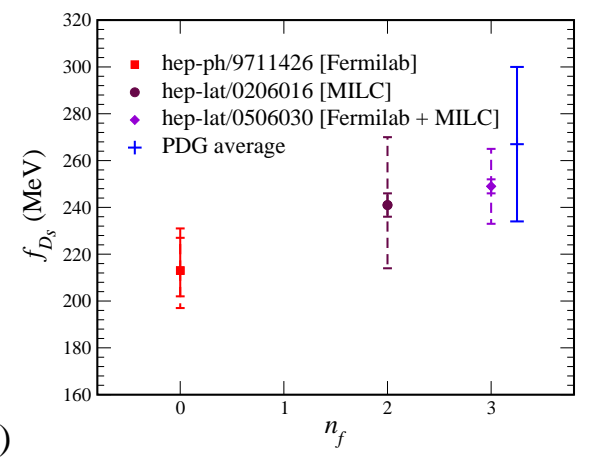

(b)

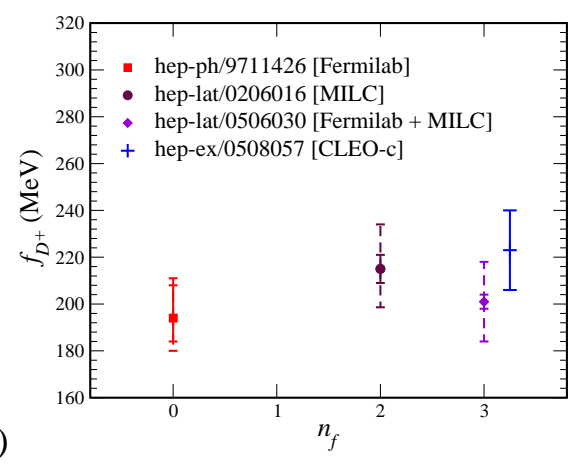

Figure 2: Dependence of (a) $f_{D_{s}}$ and (b) $f_{D^{+}}$on the number $n_{f}$ of sea flavors. Quenched $\left(n_{f}=0\right)$ [18]; $n_{f}=2[19] ; n_{f}=3$ [15]. Solid (dashed) error bars are statistical (statistical+systematic).

\section{Mass of the $B_{c}$ Meson}

The pseudoscalar $B_{c}^{+}$meson is the lowest-lying bound state of a charmed quark and a $b$ quark. CDF [20] first observed it during Run I of the Tevatron in the semileptonic decay $B_{c}^{+} \rightarrow J / \psi l^{+} v$. During Run II, DØ has confirmed the discovery in the same mode [21]. Because the neutrino is undetected, the mass resolution in semileptonic modes is poor, $\pm(300-400) \mathrm{MeV}$. Now, however, the upgraded detectors are able to reconstruct hadronic modes, such as $B_{c}^{+} \rightarrow J / \psi \pi^{+}$, which give much much better precision on $m_{B_{c}}$ [22].

At Lattice 2004 we presented results in nearly final form [23], and posted the final results on the arXiv in mid-November [24]:

$$
m_{B_{c}}=6304 \pm 12_{-0}^{+18} \mathrm{MeV},
$$

where the last error is a rough estimate of residual heavy-quark discretization effects. Soon afterwards, CDF announced a precise mass measurement. They find [25]

$$
m_{B_{c}}=6287 \pm 5 \mathrm{MeV}
$$

which agrees with Eq. (4.1) at slightly more than 1- $\sigma$.

Two comments are in order. First, the agreement at the gross level of the calculation with experiment shows that discretization effects are well under control with lattice NRQCD [27] and the 
Fermilab method [28]. Of course, this follows from the careful application of effective field theories for heavy quarks [29, 30]. Indeed, as seen in Fig. 3(a), almost no lattice spacing dependence is seen in the splitting $\Delta_{\psi \Upsilon}=m_{B_{c}}-\left(\bar{m}_{\psi}+m_{\Upsilon}\right) / 2$ that is at the crux of the calculation [26]. Moreover, it is striking how much the splitting $\Delta_{\psi}$ r changes when sea quarks are included. Figure 3(b) compares Eq. (4.1) with an old quenched calculation [26] (and the measurement [25]). The solid error bar shows the non-quenching errors, and the dashed includes the estimate of the quenching error. The inclusion of sea quarks has reduced the splitting by a factor of three or four, bringing an essentially discrepant result into agreement.

(a)

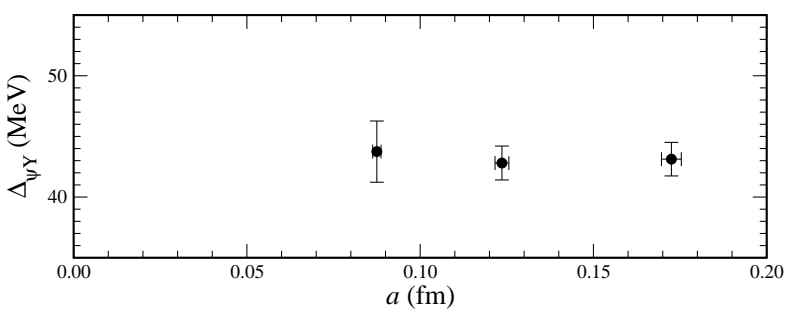

(b)

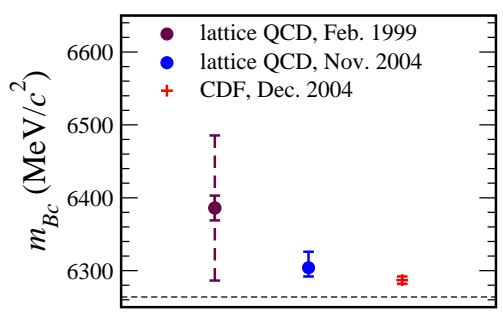

Figure 3: (a) Dependence of the splitting $\Delta_{\psi \Upsilon}$ on the lattice spacing $a$. (b) Comparison of the quenched [26], $n_{f}=2+1$ [24], and experimental [25] values of $m_{B_{c}}$; the dashed line denotes the baseline $\left(\bar{m}_{\psi}+m_{\Upsilon}\right) / 2$.

\section{Conclusions}

In the past year, three lattice-QCD calculations have been confirmed by experiment. FOCUS [10] confirmed the $q^{2}$-dependence of the $D \rightarrow K l v$ form factor [8]; CLEO-c [17] confirmed the $D$ meson decay constant [15]; and CDF [25] confirmed the mass of the $B_{c}$ meson [24]. To obtain these results it is essential to have heavy-quark discretization effects under control, as one expects from theoretical foundations [27, 28, 29, 30]. Furthermore, the comparison of quenched QCD, QCD with $2+1$ staggered flavors, and experiment shows that sea quarks are needed to obtain agreement, and that staggered quarks (in these cases) capture the needed effect.

This work has been supported in part by the U.S. National Science Foundation, the Office of Science of the U.S. Department of Energy (DOE), and the U.K. Particle Physics and Astronomy Research Council. Fermilab is operated by Universities Research Association Inc., under contract with the DOE.

\section{References}

[1] C. T. H. Davies et al. [HPQCD, MILC, and Fermilab Lattice Collaborations], Phys. Rev. Lett. 92, 022001 (2004) [hep-lat/0304004].

[2] C. Aubin and C. Bernard, Phys. Rev. D 68, 034014 (2003) [hep-lat/0304014]; ibid., 074011 (2003) [hep-lat/0306026].

[3] S. Dürr, "Theoretical issues with staggered fermion simulations," hep-lat/0509026.

[4] I. Shipsey, Nucl. Phys. Proc. Suppl. 140, 58 (2005) [hep-lat/0411009].

[5] M. Okamoto et al. [Fermilab Lattice, MILC, and HPQCD Collaborations], Nucl. Phys. Proc. Suppl. 129, 334 (2004) [hep-lat/0309107]. 
[6] M. Ablikim et al. [BES Collaboration], Phys. Lett. B 597, 39 (2004) [hep-ex/0406028].

[7] G. S. Huang et al. [CLEO Collaboration], Phys. Rev. Lett. 94, 011802 (2005) [hep-ex/0407035].

[8] C. Aubin et al. [Fermilab Lattice, MILC, and HPQCD Collaborations], Phys. Rev. Lett. 94, 011601 (2005) [hep-ph/0408306].

[9] D. Bećirević and A. B. Kaidalov, Phys. Lett. B 478, 417 (2000) [hep-ph/9904490].

[10] J. M. Link et al. [FOCUS Collaboration], Phys. Lett. B 607, 233 (2005) [hep-ex/0410037].

[11] P.B. Mackenzie et al. [Fermilab Lattice, MILC, and HPQCD Collaborations], work in progress.

[12] M. Ablikim et al. [BES Collaboration], Phys. Lett. B 610, 183 (2005) [hep-ex/0410050].

[13] G. Bonvicini et al. [CLEO Collaboration], Phys. Rev. D 70, 112004 (2004) [hep-ex/0411050].

[14] J. N. Simone et al. [Fermilab Lattice, MILC, and HPQCD Collaborations], Nucl. Phys. Proc. Suppl. 140, 443 (2005) [hep-lat/0410030].

[15] C. Aubin et al., [Fermilab Lattice, MILC, and HPQCD Collaborations], Phys. Rev. Lett. 95, 122002 (2005) [hep-lat/0506030];

J.N. Simone et al., [Fermilab Lattice, MILC, and HPQCD Collaborations], these proceedings.

[16] A. S. Kronfeld, Nucl. Phys. Proc. Suppl. 129, 46 (2004) [hep-lat/0310063].

[17] M. Artuso, talk at the XXII International Symposium on Lepton-Photon Interactions at High Energy, Uppsala, Sweden, http://lp2005.tsl.uu.se/ Ip2005/(June 30-July 5, 2005); M. Artuso et al. [CLEO Collaboration], "Improved measurement of $B\left(D^{+} \rightarrow \mu^{+} v\right)$ and the pseudoscalar decay constant $f_{D^{+}}$, , hep-ex/0508057.

[18] A. X. El-Khadra, A. S. Kronfeld, P. B. Mackenzie, S. M. Ryan, and J. N. Simone, Phys. Rev. D 58, 014506 (1998) [hep-ph/9711426].

[19] C. Bernard et al. [MILC Collaboration], Phys. Rev. D 66, 094501 (2002) [hep-lat/0206016].

[20] F. Abe et al. [CDF Collaboration], Phys. Rev. Lett. 81, 2432 (1998) [hep-ex/9805034].

[21] DØ Collaboration, DØ Note 4539-CONF (August 14, 2004);

E. Cheu [DØ Collaboration], Int. J. Mod. Phys. A 20, 3664 (2005).

[22] K. Anikeev et al., "B physics at the Tevatron: Run II and beyond," hep-ph/0201071.

[23] I. F. Allison et al. [HPQCD and Fermilab Lattice Collaborations], Nucl. Phys. Proc. Suppl. 140, 440 (2005) [hep-lat/0409090].

[24] I. F. Allison et al. [HPQCD and Fermilab Lattice Collaborations], Phys. Rev. Lett. 94, 172001 (2005) [hep-lat/0411027].

[25] D. Acosta et al. [CDF Collaboration], "Evidence for the exclusive decay $B_{c}^{ \pm} \rightarrow J / \psi \pi^{ \pm}$and measurement of the mass of the $B_{c}$ meson," hep-ex/0505076.

[26] H. P. Shanahan, P. Boyle, C. T. H. Davies, and H. Newton [UKQCD Collaboration], Phys. Lett. B 453, 289 (1999) [hep-lat/9902025].

[27] G. P. Lepage and B. A. Thacker, Nucl. Phys. Proc. Suppl. 4, 199 (1987);

B. A. Thacker and G. P. Lepage, Phys. Rev. D 43, 196 (1991).

[28] A. X. El-Khadra, A. S. Kronfeld, and P. B. Mackenzie, Phys. Rev. D 55, 3933 (1997)

[hep-lat/9604004].

[29] G. P. Lepage et al., Phys. Rev. D 46, 4052 (1992) [hep-lat/9205007].

[30] A. S. Kronfeld, Phys. Rev. D 62, 014505 (2000) [hep-lat/0002008]. 